\title{
Ungeduld der Erkenntnis
}

Eine klischeewidrige Festschrift für Hubert Orłowski

Herausgegeben von Włodzimierz Bialik, Czesław Karolak und Maria Wojtczak

Redaktionelle Mitarbeit: Jan-Moritz Werk 
Bibliografische Information der Deutschen Nationalbibliothek

Die Deutsche Nationalbibliothek verzeichnet diese Publikation

in der Deutschen Nationalbibliografie; detaillierte bibliografische

Daten sind im Internet über http://dnb.d-nb.de abrufbar.

Wydano $\mathrm{z}$ finansowym wsparciem Fundacji Współpracy Polsko-Niemieckiej.

Herausgegeben mit finanzieller Unterstützung der Stiftung für Deutsch-Polnische Zusammenarbeit.

Gedruckt auf alterungsbeständigem, säurefreiem Papier.

ISBN 978-3-631-64341-9 (Print)

E-ISBN 978-3-653-03333-5 (E-Book)

DOI 10.3726/978-3-653-03333-5

(c) Peter Lang $\mathrm{GmbH}$

Internationaler Verlag der Wissenschaften

Frankfurt am Main 2014

Alle Rechte vorbehalten.

Peter Lang Edition ist ein Imprint der Peter Lang GmbH.

Peter Lang - Frankfurt am Main · Bern · Bruxelles · New York Oxford $\cdot$ Warszawa $\cdot$ Wien

Das Werk einschließlich aller seiner Teile ist urheberrechtlich geschützt. Jede Verwertung außerhalb der engen Grenzen des

Urheberrechtsgesetzes ist ohne Zustimmung des Verlages unzulässig und strafbar. Das gilt insbesondere für

Vervielfältigungen, Übersetzungen, Mikroverfilmungen und die Einspeicherung und Verarbeitung in elektronischen Systemen.

www.peterlang.com

\section{Inhaltsverzeichnis}

Anstelle einer Einleitung

Whodzimierz Bialik

Aufzug 1

Mein Lebenslauf mit Hubert Orłowsk

Czeslaw Karolak

Aufzug 2

System recovery als Metapher?

Maria Wojtczak

Aufzug 3

liber amicorum

Edward Bialek

"Ein Nationalsozialist des Herzens": Peter Martin Lampels Aufstieg und Fall im Dritten Reich in Selbstzeugnissen und Dokumenten

Włodzimierz Bialik

Der blinde Seher oder Der erste Jonas-Vogel-(Kriminal-)Roman

von Friedrich Ani.Ein offener Brief an Herrn Professor Orłowski

Hubertus Fischer

„Warte Bonaparte, warte..." Fontane und die Völkerschlacht 1813

Michat Glowinski

Wie wurde deutsche Musik unmittelbar nach dem Zweiten Weltkrieg

in Polen gehört?

Joanna Jablkowska

„... die Worte// Fallen in das Getriebe der Welt uneinholbar // Kenntlich machend die Dinge oder unkenntlich". Heiner Müllers Konzept

der Tragödie

Jürgen Joachimsthaler

„Polnische Wirtschaft" $\cap$ Bayerische Typenkomödie

Zur Schnittmenge zwischen preußischem Bayern- und preußischem

Polenbild 
Jerzy Katażny

„Warschau - eine merkwürdige Stadt“. Zur Topographie der Erinnerung an den Krieg und die Judenvernichtung in der polnischen Prosa

der Gegenwart.

Czestaw Karolak

Digitale Wiedergeburten für den Tempel der Weisheit.

Bibliotheks- und museale Sammlungen im Netz als Antwort

auf die Herausforderungen der Informationsgesellschaft.

\section{Maria Klańska}

Alexander Granach im Exil. Das Zeitgeschehen in den Augen

des Schauspielers anhand seiner Briefe an Lotte Lieven.

\section{Jïrgen Kocka}

Arbeitergeschichte. Thr Niedergang und ihre Wiederbelebung im Zeichen

der Globalgeschichte.

Kornelia Kończal

Das Schreiben und das Schweigen über die Plünderung des deutschen

Eigentums. Die identitätsstiftende Figur des szabrownik

im Nachkriegspolen

Andreas Lawaty

Korrespondenzen und Divergenzen Karl Dedecius und ,seine“" Autoren

Eukasz Musial

Lexikon der polnischen Schriftsteller. Allenstein 2014

Henryk Olszewski

Laudatio für eine wichtige Editionsinitiative: Fünfunddreißig Bände

Posener Deutsche Bibliothek

Stawomir Piontek

Playing History oder was suchen Erinnerungskulturen

in den Computerspielen? Ein kontextualisiertes Forschungsdesiderat

Andrzej Sakson

Ermländer und Masuren in der Postmigrationsgesellschaft.
Heinz Schilling

Religion und Gewalt zu Beginn der europäischen Neuzeit -

Was können wir aus dem europäischen Konfessinsfundamentalismus

des 16. und 17. Jahrhunderts lernen?

Karl Schlögel

Unterwegs in unsichtbare Städte. Europa molekular.

Peter Steinbach

Das Jahrhundert der Diktatoren - auch ein Jahrhundert des Widerstands........263

Robert Traba

"Offener Regionalismus" - eine praktische Bürgerphilosophie:

In memoriam Lew Kopelew (1912-1997)

Maria Wojtczak

Eine unverbindliche Heimat ist keine Heimat. Die Generation

der Posener Deutschen

Marek Zybura

Dudeczku mój drogi... Droga Alice...Zu einer gewissen amitié amoureuse

à trois in der Geschichte der deutschen Rezeption des Werks

von Witold Gombrowicz

Leszek Żylinski

Die Geburt der neuen Demokratie aus dem Geist Europas.

Zu den Europa-Essays von Jürgen Habermas und Robert Menasse 


\title{
Playing History oder was suchen Erinnerungskulturen in den Computerspielen? Ein kontextualisiertes Forschungsdesiderat
}

\author{
Slawomir Piontek
}

Poznań

Computerspiele als ein kulturelles Phänomen werden noch vielerorts für ein ,unseriöses' Medium gehalten, das für Jugendliche bestimmt ist, mit dem man die Zeit totschlägt und das lediglich individuelle und soziale Probleme verursacht. Aber schon ein flüchtiger Blick auf die große Differenzierung des Lebensalters In der Spielergruppe, das riesige thematische und gattungsmäßige Angebot der Spiele und die Milliardenumsätze in dieser Wirtschaftsbranche führt zur Reflexion, dass die Faszination für Computerspiele keine Randerscheinung und weder vorübergehend noch ausschließlich auf die Jugend bezogen ist. Als möglicher Gegenstand kulturwissenschaftlicher Forschung werden Computerspiele ebenfalls stark beargwöhnt, die Analysen konzentrierten sich in der ersten Phase des Forschungsinteresses vor allem auf die oben genannten negativen Aspekte. In den 1980er und 1990er Jahren untersuchte man u.a. den Einfluss der Spiele auf das Sozialverhalten und auf die Wertvorstellungen der Jugend, wobei nicht selten die Erhebungen als Beweis für die vorformulierte These vom rassistischen, sexistischen, Gewalt glorifizierenden, süchtig machenden, soziale Isolation bewirkenden usw. Charakter der Spiele herangezogen wurden. Forschungen, die diesem „Besorgnis-Diskurs"1 zuzuschreiben wären, werden bis heute fortgesetzt. ${ }^{2}$

I Jutta Röser (2005): „Gewaltdarstellung«. In: Siegfried Weischenberg/ Hans J. Kleinsteuber/ Bernhard Pörksen (Hg.): Handbuch Journalismus und Medien. Konstanz: UVK, S. $82-86$, hier. S. 82.

2 Vgl ua Horst W Opaschowski (1983): Arbeit, Freizeit, Lebenssinn? Orientierungen für eine Zukunft, die längst begonnen hat, Opladen: Leske+Budrich; Wolfgang Poeplau (1992): Monster, Macht und Mordmaschinen: Computeppiele: Digitale Illusionen und soziale Wirklichkeit, Wuppertal: Hammer, Ullich Dittler (1993): Software statt Teddybär: Computerspiele und die pädagogische Auseinandersetzung. München: E. Reinhardt; Rainer Osenberg (1993): Götter, Geld und große. Taten: Strategische Simulationen auf dem Computer: Lösungshinweise zu den Top-Spielen, Düsseldorf: Sybex; Aleksandra Gała Iwona Ulfik (Hg.) (2000): Oddziaływanie agresywnych gier komputerowych na psychikę dzieci. Lublin: Wydawnictwo Artom; Maria Braun-Gałkowska; Iwona Ulfik-Jaworska (2002): Zabawa w zabijanie. Oddziaływanie przemocy prezentowanej w mediach na psychikę dzieci. Lublin: Wyd. Gaudium; Iwona Ulfik-Jaworska (2005): Komputerowi mor- 
Das Interesse für historische Inhalte in den Computerspielen konzentrierte sich anfänglich auf die Frage der Einsetzbarkeit der Spiele als didaktisches Hilfsmittel in der Geschichtsdidaktik. ${ }^{3}$ Problematisch ist dieser Ansatz insofern, als dass die Konstruktions-, Spiel- und Marktregeln, die dieses Medium bestimmen, wenig gemeinsam mit den Voraussetzungen der Didaktik haben. Der entscheidende Paradigmenwechsel Ende des letzten Jahrzehnts war die Folge des steigenden Interesses für den Übergang ,von der Visualisierung zur Virtualisierung des Erinnerns ${ }^{* 4}$ und für Repräsentationformen der Geschichte in den populären Medien (History goes pop ${ }^{5}$ ). In diesen Bereich geraten auch Computerspiele, wobei man anmerken muss, dass dieser Prozess gerade jetzt beginnt und an Dynamik gewinnt und die Analysen selektiv und partiell sind. Hingewiesen werden soll hier auf methodologische Postulate und typologische Vorschläge von Angela Schwarz, die historische Computerspiele als kulturelle Artefakte versteht, die nach eigenen spezifischen Regeln funktionieren und die auch eigene Perspektiven auf die (Pop) Geschichte generieren können. ${ }^{6}$

Innerhalb der seit 1981 erschienenen 2009 Historiespiele beziehen sich $26 \%$ der Spiele auf den Zweiten Weltkrieg. ${ }^{.}$Dieses intensive Interesse sowohl auf der Seite der Produzenten als auch der Nutzer hat nachvollziehbare Gründe. Es legt zugleich die Notwendigkeit nahe, diese Erscheinung einer komplexen Untersu-

dercy. Tendencje konstruktywne i destruktywne u dzieci grających w „agresywne” gry komputerowe. Lublin: Wydawnictwo KUL; Hartmut Gieselmann (2002): Der virtuelle Krieg: Zwischen Schein und Wirklichkeit im Computerspiel, Hannover: Offizin; Rudolf und Renate Hänsel (Hg.) (2005): ,Da spiel ich nicht mit!' Auswirkungen von ,Unterhaltungsgewalt' in Fernsehen, Video- und Computerspielen - und was man dagegen tun kann: Eine Handreichung für Lehrer und Eltern, Donauwörth: Auer; Stefan Gesmann (2006): ,Friendly Fire‘ im Kinderzimmer, Saarbrücken: Müller; Sabine Geyer (2006): Computerspiele, Gewalt und Terror Management: Grundlagen, Theorie, Praxis, Saarbrücken: Müller.

3 Z. B. Waldemar Grosch (2002): Computerspiele im Geschichtsunterteicht, Schwalbach am Taunus: Wochenschau Verlag; Jeremiah B. McCall (2011): Gaming the past. Using video games to teach secondary history. New York: Routledge.

4 Claus Leggewie (2009): Zur Einleitung: Von der Visualisierung zur Virtualisierung der Erinnerns. In: Erik Meyer (Hg.): Erinnerungskultur 2.0. Kommemorative Kommunikation in digitalen Medien. Frankfurt/Main, New York,: Campus-Verl., s. 9-28.

5 Barbara Korte, Sylvia Paletschek (2009): History goes pop. Zur Repräsentation von Geschichte in populären Medien und Genres. Bielefeld: transcript.

6 Angela Schwarz (2009): "Wollen sie wirklich nicht weiter versuchen, diese Welt zu dominieren«: Geschichte in Computerspielen. In: Barbara Korte, Sylvia Paletschek (2009): History goes pop. Zur Repräsentation von Geschichte in populären Medien und Genres. Bielefeld: transcript, S. 313-340; Angela Schwarz (2010) (Hg.): 'Wollten Sie auch immer schon einmal pestverseuchte Kühe auf Ihre Gegner werfen?": Geschichte im Computerspiel. Berlin: Lit.

7 Schwarz (2010), S. 15. chung zu unterziehen. Die Perspektive, die in dieser Hinsicht als am geeignetsten erscheint, ist die Analyse der Computerspiele zum Zweiten Weltkrieg als Medien des kulturellen Gedächtnisses, mit besonderer Berücksichtigung der Strategien der Wirklichkeitskonstruktionen der 'gespielten Welt'.

Wie gesagt, werden Computerspiele neben anderen visuellen Medien (Film, Fernsehen, Internet) ein immer wichtigeres Element der medialen Sozialisierung der Gesellschaft und - hinsichtlich der hier zur Debatte stehenden Problematik eines der wichtigsten Medien der Präsenz und der Präsentation der Geschichte im öffentlichen Raum. Die Konstruktion historischer Bilder, darunter auch der Bilder vom Zweiten Weltkrieg, unterliegt natürlich allen Einschränkungen, die mit dem jeweiligen Medium verbunden sind (u. a. Raum- und Zeitstruktur, Grafik-Engine, Programmiersprachen, Erwartung eines positiven Ergebnisses usw.), die das finale Bild der dargestellten Welt so stark determinieren, dass die Frage nach ihrer Adäquatheit oder Kompatibilität mit der historischen Faktografie und geschichtswissenschaftlichem Verständnis der Prozesshaftigkeit als unmotiviert oder wenigstens problematisch erscheint. ${ }^{8}$ Durchaus berechtigt ist aber eine andere Voraussetzung: nimmt man an, dass Computerspiele ein gleichberechtigtes Medium der Transmission der Kulturbilder sind und außerdem ein immer prominenteres Medium der Erinnerungskulturen, so muss man folgerichtig akzeptieren, dass die Spiele eigene historische Narrative und Vergangenheitsbilder kreieren.

Das Kundeninteresse an Computerspielen mit historischen Inhalten, darunter auch an den Spielen über den Zweiten Weltkrieg, wächst ständig seit Mitte der 1990er Jahre, was sehr gut an den Neuerscheinungen und Verkaufszahlen abzulesen ist." Zum einen ist dies durch die sehr schnell steigende technologische Leistungsfähigkeit dieses Mediums begünstigt, die immer neuere und attraktivere Narrations- und Expressionsformen bietet (viele neue Impulse hat hier z.B. der narrativ-ikonographische Paradigmenwechsel in der filmischen Kriegsdarstellung seit „Saving Private Ryan“ (1997) eingebracht), zum anderen resultiert das Interesse für die Zeit des Zweiten Weltkrieges aus der besonderen Position dieses Ereignisses auf der Zeitachse zwischen dem direkten, nicht modifizierbaren kommunikativen und dem intentionalen, plastischen kulturellen Gedächtnis. Ein solcher chronologischer Status dieses Großereignisses bietet besondere Möglichkeiten, seine Geschichte(n) zu erzählen.

Die Analyse dieser Geschichten, Bilder und Narrative in den Computerspielen kann im Kontext der Positionierung der Spiele als Medien einer populären

8 Vgl. etwa Thomas Kubetzky (2012): Computerspiele als Vermittlungsinstanzen von Geschichte? Geschichtsbilder in Aufbausimulationsspielen am Beispiel von CIVILIZATION IIJ. In: Angela Schwarz (Hg.): 'Wollten Sie auch immer schon einmal pestverseuchte Kühe auf Ihre Gegner werfen?': Geschichte im Computerspiel. 2. Aufl. Berlin: Lit, S. 94.

9 Schwarz (2010), S. 1 I. 
Erinnerungskultur erfolgen. Daraus resultiert die Frage nach den Strategien und Mechanismen der Konstruktion dieser Bilder und Narrative. Ansätze, die sich aus dieser Kontextualisierung ergeben, können in unterschiedliche Richtungen gehen: Nimmt das Gedächtnis des Zweiten Weltkrieges einen universalistischen Charakter an oder enthält es auch ,nationale Konzessionen'? Inwiefern ist es ein europäisches, oder sogar ein transatlantisches Gedächtnis und inwiefern ist es national diversifiziert? Gibt es Modifizierungen der Spiele oder Einschränkungen des Vertriebs auf den nationalen Märkten und -wenn ja - welche sind dies? Variiert und erweitert man diese Frage: Inwiefern spiegeln diese Spiele den gegenwär tigen Macht- und Ideologiediskurs wider? Ist die Thematisierung des Zweiten Weltkrieges als ein identitätskonsolidierendes Element für moderne Menschen westlicher Zivilisation intendiert?

Die Frage nach den Möglichkeiten, durch Spiele Geschichte(n) zu erzählen betrifft nicht nur das Was, d.h. welche Geschichte(n) der Zweiten Weltkrieges erzählt werden, sondern auch das Wie, also welche narrativen Strategien sich in den Computerspielen im Prozess der Virtualisierung des kulturellen Gedächtnisses des II. WK als die wirksamsten erweisen.

Eine weitere Kontextualisierung und Erweiterung des Problemfeldes kann unter Heranziehung der Hersteller und der Spielerszene erfolgen. Spiele können als offene Texte verstanden werden, die sich während des Spielens, also bei der Handhabung durch Spieler konkretisieren. Dies bewirkt eine Spannung zwischen den Bedeutungen, die durch die Hersteller implementiert wurden und jenen, die spontan durch die Spieler konstruiert werden. Diese Spannung mündet in eine grundsätzliche Opposition zwischen dem globalen Charakter der Spielindustrie und der lokalen Aufnahme der Spiele, deren Charakter häufig länderbezogen ist Das wirft weitere Fragen auf, etwa nach der Aktivität der Spieler, ihre lokalen Gedächtnisse zu mobilisieren und sie dem globalen Diskurs entgegenzusetzen. Eine andere Facette des gleichen Problems sind die Lokations- und Vertriebsstrategien, die durch global agierende Hersteller verfolgt werden.

Eine solche Formulierung der Forschungsfragen determiniert die Wahl methodologischer Zugänge. Rein geschichtswissenschaftliche Ansätze, die nach Repräsentationsformen der Vergangenheit in Historiespielen fragen, kommen zum Ergebnis, dass die durch die Hersteller häufig mit Nachdruck herausgestrichene ,historische Exaktheit' der Spiele sich schließlich doch an den Spielregeln des Marktes und den Spielerwartungen der Nutzer orientiert. Vereinfachungen, Verkürzungen, Umkehrungen von Ursache und Wirkung oder anachronistische Projektionen zu erwartender Vorstellungen moderner User auf das konstruierte $\mathrm{Ge}$ schichtsbild sind daher nicht als Kunstfehler aufzufassen, sondern als Ergebnis der Spielregeln innerhalb der Spielproduktion.
Unabhängig vom Grad der Sättigung mit historischen Details liefern die Spiele vor allem aber fiktionale Geschichten, die als solche untersucht werden sollten. Eine Überprüfung der historischen Korrektheit einer fiktionalen Geschichte (im Text, Film) kann als interpretatorisches Beiwerk herangezogen werden, sie sagt aber weder etwas vom Charakter noch von der Funktion der übermittelten Geschichtsbilder aus, denn in fiktionalen Geschichten (hier im Gewand populärer Geschichtsbilder) haben historische Unstimmigkeiten bzw. „Illusionen von Historizität $t^{510}$ den gleichen semantischen Wert wie die ,stimmigen" Momente. Was also in methodologischer Hinsicht nottut, um der strukturellen und semantischen Beschaffenheit der ,gespielten Welt' in den Historiespielen auf die Spur zu kommen, ist eine geschickte Mischung aus Hermeneutik, Methodologie populärerer Geschichtsnarrativik und Spielforschungstheorien (E. Aarseth, J. Juul "). Für Spiele, die thematisch auf den Zweiten Weltkrieg rekurrieren, besonders fruchtbar zeigen sich Ansätze innerhalb der Theorien der Erinnerungskulturen und der kollektiven Gedächtnisse, wie sie von Maurice Halbwachs formuliert und von Pierre Nora oder Jan und Aleida Assmann weiterentwickelt wurden. Denn nicht die wissenschaftliche Exaktheit, sondern das Potenzial, gesellschaftlich wirkmächtige Vorstellungen und Konstruktionen von Geschichte zu kreieren und zu übermitteln, also die mediale Repräsentanz der Geschichte in den Computerspielen verdient Aufmerksamkeit, sprich: eine neue interdisziplinäre Lesart. Der erinnerungskulturelle Ansatz ist in Bezug auf II.WK-Spiele besonders legitimiert: Die gegenwärtige spezifische chronologische ,Lagerung" des Zweiten Weltkrieges bewirkt, dass diese vergangenen Ereignisse immer noch konkreten Einfluss auf gegenwärtige soziale und kulturelle Prozesse ausüben können (etwa die letzten Kontroversen um die Waffen-SS- oder NSDAP-Zugehörigkeit prominenter Gestalten des deutschen politischen oder kulturellen Lebens ${ }^{12}$ oder den Skandal um den Kunstraub ${ }^{13}$ ), die Perspektive des ,virtuellen Erinnerns ${ }^{6 / 4}$ kann aber auch mit

10 Thomas Kubetzky (2012): Computerspiele als Vermittlungsinstanzen von Geschichte? Geschichtsbilder in Aufbausimulationsspielen am Beispiel von CIVILIZATION III. In: Angela Schwarz (Hg.): 'Wollten Sie auch immer schon einmal pestverseuchte Kühe auf Ihre Gegner werfen?‘: Geschichte im Computerspiel. 2. Aufl. Berlin: Lit, S. 77.

11 Espen J. Aarseth (2004): Playing Research: Methodological approaches to game analysis, http://www.cs.uu.nl/docs/vakken/vw/literature/02.GameApproaches2.pdf; Jesper Juul (2005): Half-Real: Video Games between Real Rules and Fictional Worlds, Cambridge, Massachusetts: MIT Press.

12 Malte Herwig (2013): Die Flakhelfer: Wie aus Hitlers jüngsten Parteimitgliedern Deutschlands führende Demokraten wurden. München: Deutsche Verlags-Anstalt.

I3 http://www.spiegel.de/kultur/gesellschaft/nazi-raubkunst-bilder-von-picasso-matisse-nolde-in-muenchen-entdeckt-a-931507.html

14 Steffen Bender (2012): Virtuelles Erinnern. Kriege des 20. Jahrhunderts in Computerspielen. Bielefeld: Transcript. 
Erfolg auf thematisch anders ausgerichtete Spiele bezogen werden. ${ }^{15}$ Analysen, die ein ähnliches Profil aufweisen, finden sich auch bei Gunnar Sandkühler ${ }^{16}$. Einen anderen Ansatz, der sich jedoch auf das gleiche methodologische Paradigma bezieht, liefert Tobias Bevc in der Analyse za politischen und sozialen Strukturen in Computerspielen. ${ }^{17}$

Die Ergebnisse einer solchen komplexen Analyse der Konstruktion der Wirklichkeit des Zweiten Weltkrieges in den Computerspielen ermöglichen ein besseres Verständnis der Transformationsprozesse der gegenwärtigen geschichtlichen Identität im interkulturellen Raum und gewähren einen Einblick in das Angebot eines jungen Mediums, das aktiv und massenhaft kollektive Vorstellungen von der Vergangenheit gestaltet.

\section{Literatur}

Anderson, Benedict (2006): Imagined communities. Reflections on the origin and spread of nationalism. London: Verso.

Assmann, Aleida (2007): Geschichte im Gedächtnis. Von der individuellen Erfahrung zur öffentlichen Inszenierung. München: Beck.

Assmann, Jan (2006): Das kulturelle Gedächtnis Schrift, Erinnerung und politische Identität in frühen Hochkulturen. München: Beck.

Aarseth, Espen J (2004): Playing Research: Methodological approaches to game analysis, http://www.cs.uu.nl/docs/vakken/vw/literature/02.GameApproaches2.pdf.

Bender, Steffen (2010): Durch die Augen einfacher Soldaten und nameloser Helden. Weltkriegsshooter als Simulation historischer Kriegserfahrung? In: Schwarz, Angela (2010) (Hg.): 'Wollten Sie auch immer schon einmal pestverseuchte Kühe auf Ihre Gegner werfen?': Geschichte im Computerspiel. Berlin: Lit, S. 137-162.

Bender, Steffen (2012): Virtuelles Erinnern. Kriege des 20. Jahrhunderts in Computerspielen. Bielefeld: Transcript.

Bevc, Tobias (2007) (Hg.): Computerspiele und Politik: Zur Konstruktion von Politik und Gesellschaft in Computerspielen. Berlin: Lit.

Gesmann, Stefan (2006): ,Friendly Fire' im Kinderzimmer. Saarbrücken: Müller.

Geyer, Sabine (2006): Computerspiele, Gewalt und Terror Management: Grundlagen, Theorie, Praxis. Saarbrücken: Müller.

15 Ebd.

16 Gunnar Sandkühler (2009): Der Zweite Weltkrieg im Computerspiel: Ego-Shooter als Geschichtsdarstellung zwischen Remediation und Immersion. In: Erik Meyer (Hg.): Erinnerungskultur 2.0. Kommemorative Kommunikation in digitalen Medien. Frankfurt/Main, New York, NY: Campus-Verl., S. 55-65.

17 Tobias Bevc (2007) (Hg.): Computerspiele und Politik: Zur Konstruktion von Politik und Gesellschaft in Computerspielen, Berlin: Lit.
Gieselmann, Hartmut (2002): Der virtuelle Krieg: Zwischen Schein und Wirklichkeit im Computerspiel. Hannover: Offizin.

Grosch, Waldemar (2002): Computerspiele im Geschichtsunterreicht. Schwalbach am Taunus: Wochenschau Verlag.

Halbwachs, Maurice (1991): Das kollektive Gedächtnis. Frankfurt/Main: Fischer.

Hänsel, Rudolf und Renate (Hg.) (2005): ,Da spiel ich nicht mit!' Auswirkungen von ,Unterhaltungsgewalt in Fernsehen, Video- und Computerspielen - und was man dagegen tun kann: Eine Handreichung für Lehrer und Eltern. Donauwörth: Auer.

Herwig, Malte (2013): Die Flakhelfer: Wie aus Hitlers jüngsten Parteimitgliedern Deutschlands führende Demokraten wurden. München: Deutsche Verlags-Anstalt.

Juul, Jesper (2005): Half-Real: Video Games between Real Rules and Fictional Worlds. Cambridge, Massachusetts: MIT Press.

Korte, Barbara; Paletschek, Sylvia (2009) (Hg.): History goes pop. Zur Repräsentation von Geschichte in populären Medien und Genres. Bielefeld: transcript.

Leggewie, Claus (2009): Zur Einleitung: Von der Visualisierung zur Virtualisierung der Erinnerns. In: Meyer, Erik (Hg.): Erinnerungskultur 2.0. Kommemorative Kommunikation in digitalen Medien. Frankfurt/Main, New York, NY: Campus-Verl., S. 9-28.

McCall, Jeremiah B. (2011): Gaming the past. Using video games to teach secondary history New York: Routledge.

Nora, Pierre (2001) (Hg.): Zwischen Geschichte und Gedächtnis. Frankfurt/Main: Fischer

Oittler, Ulrich (1993): Software statt Teddybär: Computerspiele und die pädagogische Auseinandersetzung. München: E. Reinhardt.

Opaschowski, Horst W. (1983): Arbeit, Freizeit, Lebenssinn? Orientierungen für eine Zukunft, die längst begonnen hat. Opladen: Leske+Budrich.

Osenberg, Rainer (1993): Götter, Geld und große Taten: Strategische Simulationen auf dem Computer: Lösungshinweise zu den Top-Spielen. Düsseldorf: Sybex.

Poeplau, Wolfgang (1992): Monster, Macht und Mordmaschinen: Computeppiele: Digitale I1lusionen und soziale Wirklichkeit. Wuppertal: Hammer.

Röser, Jutta (2005): „Gewaltdarstellung«. In: Siegfried Weischenberg/ Hans J. Kleinsteuber/ Bernhard Pörksen (Hg.): Handbuch Journalismus und Medien, Konstanz: UVK, S. 82-86.

Sandkühler, Gunnar (2009): Der Zweite Weltkrieg im Computerspiel: Ego-Shooter als Geschichtsdarstellung zwischen Remediation und Immersion. In: Meyer, Erik ( $\mathrm{Hg}$.): Erinnerungskultur 2.0. Kommemorative Kommunikation in digitalen Medien. Frankfurt/Main, New York, NY: Campus-Verl., S. 55-65.

Schwarz, Angela (2010) (Hg.): 'Wollten Sie auch immer .schon einmal pestverseuchte Kühe auf Ihre Gegner werfen?‘: Geschichte im Computerspiel. Berlin: Lit.

Schwarz, Angela (2009): "Wollen sie wirklich nicht weiter versuchen, diese Welt zu dominieren«: Geschichte in Computerspielen. In: Korte, Barbara; Paletschek, Sylvia: History goes pop. Zur Repräsentation von Geschichte in populären Medien und Genres. Bielefeld: transcript, S. 313-340. 